\section{$\underset{\substack{\text { hommes } \\ \text { \& migrations }}}{ }$}

\section{Hommes \& migrations}

Revue française de référence sur les dynamiques

migratoires

Les Turcs en France : quels ancrages?

\title{
Jeunes générations turques de France et d'Allemagne
}

Des expériences plurielles

\section{Maïtena Armagnague}

\section{CpenEdition}

\section{Journals}

Édition électronique

URL : http://journals.openedition.org/hommesmigrations/297

DOI : 10.4000/hommesmigrations.297

ISSN : 2262-3353

Éditeur

Musée national de l'histoire de l'immigration

Édition imprimée

Date de publication : 1 juillet 2009

Pagination : 50-60

ISSN : 1142-852X

\section{Référence électronique}

Maïtena Armagnague, « Jeunes générations turques de France et d'Allemagne », Hommes \& migrations [En ligne], 1280 | 2009, mis en ligne le 29 mai 2013, consulté le 10 décembre 2020. URL : http:// journals.openedition.org/hommesmigrations/297 ; DOI : https://doi.org/10.4000/hommesmigrations. 297 


\section{Jeunes générations turques de France et d'Allemagne Des expériences plurielles}

Par Maïtena Armagnague, doctorante, ATER en sociologie, université de Bordeaux 2

En France comme en Allemagne, l'identité des jeunes d'ascendance turque n'est pas homogène. Elle est le fruit d'un jeu, à chaque fois recommencé, entre la communauté d'origine et la société majoritaire. Suivant le résultat de ces rapports de force, ce sont la communauté ethnique, l'assimilation culturelle ou l'intégration économique qui priment. De Bordeaux à Hambourg, les jeunes d'origine turque tentent de façonner leurs repères à la croisée d'univers de valeurs parfois contradictoires. 
En France et en Allemagne, les jeunes issus de l'immigration sont souvent au cceur des questions urbaines, identitaires et d'insertion professionnelle ${ }^{(1)}$, car ils matérialisent l'efficacité des dispositifs d'intégration et de résolution de la question sociale. Les générations nées dans les sociétés majoritaires ont été socialisées par les institutions nationales, mais leur évocation en tant que "problème" révèle la partialité de leur inscription sociale. En Allemagne, les jeunes d'ascendance turque ont été pionniers dans l'appréhension de l'immigration contemporaine comme une question problématique (die türkische Frage). En France, les jeunes issus des migrations postcoloniales ont préalablement occupé cette place et sont encore victimes de discriminations. Cela étant, en France aussi, l'immigration turque pose question $^{(2)}$. Trop souvent, les sociétés majoritaires essentialisent les difficultés d'intégration subies par les populations turques et renvoient sur ces dernières la responsabilité de leur condition sociale : les Turcs seraient communautaristes, formeraient des sociétés parallèles (Parallelgesellschaft). Il s'agit d'une manière subtile d'ignorer les formes actuelles de la question sociale, l'ethnicité et le paupérisme juvénile urbain. Néanmoins, il existe des réseaux turcs structurés organisant l'espace ethnique ${ }^{(3)}$. Ces réseaux associatifs, religieux, économiques transnationaux ${ }^{(4)}$, valent à l'immigration turque la dénomination d'immigration diasporique et communautaire ${ }^{(5)}$. L'expérience sociale des jeunes d'ascendance turque est donc le fruit de la rencontre entre une société nationale, un contexte urbain local et une population immigrée, réputée communautaire.

\section{La complexité de l'expérience migratoire}

Aux États-Unis puis en France, les théories de l'assimilation segmentée ${ }^{(6)}$ ont démontré l'hétérogénéité des vagues migratoires. L’observation des expériences concrètes des jeunes issus de l'immigration confirme qu'il est nécessaire de sortir d'une vision linéaire de l'intégration sociale menant à l'assimilation. L'inscription des individus dans la société (et les productions subjectives en découlant) est toujours fonction des modalités d'acculturation et d'intégration économique à la société dominante, aux standards de la classe moyenne. L'approche de François Dubet séparant le processus d'intégration, soulignant les strictes dimensions économiques du fait migratoire, de l'assimilation, en désignant les aspects exclusivement culturels, offre l'avantage de postuler la dissonance entre ces deux notions ${ }^{(7)}$. La pluralité des expériences des jeunes d'origine turque nécessite une telle grille d'analyse. Plusieurs configurations résultent de la nature des relations que les jeunes entretiennent avec la communauté ethnique, la société et ses institutions, et la 
culture juvénile urbaine de leurs quartiers de résidence. Les conditions sociales quotidiennes de ces derniers produisent des identités et des subjectivités particulières. Trois types différents de rapport à soi sont observables à Hambourg et à Bordeaux : une figure libérale de placement, légitimiste et conformiste ; une figure minoritaire réactive, complexe et ambivalente dans ses injonctions de justice sociale; et enfin une posture identitaire ethnique plus revendicative. Ces constructions sociales sont présentes dans les deux pays, mais s'y déclinent différemment du fait des inflexions dues aux contextes locaux et institutionnels.

\section{Un positionnement libéral et conformiste}

En France et en Allemagne s'observe une dynamique migratoire classique (conforme au processus linéaire d'assimilation), individuelle et libérale. Certains jeunes s'intègrent et s'assimilent en effet dans les espaces français et allemand et se rapprochent du standard de réussite des sociétés d'implantation. Ils partagent les normes et les valeurs de mobilité sociale, de consommation, utilisent la langue légitimée par la société environnante et s'éloignent souvent de la communauté ethnique d'origine. Leur positionnement à l'égard des institutions est volontariste, ce qui est rétribué par un encouragement et une valorisation émanant de ces dernières (école, espace politique, médias). En France et en Allemagne, les jeunes inscrits dans cette dynamique ont souvent de très bons résultats scolaires. Studieux et confiants dans l'école, ils ont souvent des parents qui les accompagnent dans leur parcours scolaire.

"L'école a toujours été très importante pour moi et je me souviens que certains profs ont vraiment beaucoup fait pour moi [...]. Je me rappelle une maîtresse en primaire, elle était super, elle prenait du temps pour me parler comme jétais Turque, elle rencontrait mes parents, elle était très gentille [...]. Elle téléphonait même chez mes parents le soir pour les conseiller..." (Yasmin,18 ans, étudiante, Bordeaux)

Dans ces parcours, la possession de ressources scolaires autorise le détachement de la communauté d'origine, notamment pour l'accès à l'emploi. Le registre méritocratique et la foi en son propre exemple de self-made man sont souvent mis en avant et s'appuient parfois sur une sévérité à l'égard des modes de vie moins conformes à la norme dominante de réussite.

Ces jeunes ne se distinguent plus formellement des jeunesses française et allemande. Leurs relations et leurs pratiques sociales s'inscrivent dans des espaces non turcs et se caractérisent par leur caractère libéral et individualiste. Garçons et filles expliquent qu'ils sortent et sont totalement intégrés dans les espaces juvéniles non turcs 
de socialisation (soirées étudiantes, par exemple). Leurs flirts amoureux sont spontanés et laissent place à l'imprévu. Certains vivent seuls en appartement ou en colocation et le justifient comme voie d'accès à leur liberté, calquée sur la norme ambiante. "J'ai pris un appartement seule ; au début, c'était difficile de le faire accepter à mes parents, mais comme je faisais des études, ils m’ont laisséfaire et ils ont vu quillspouvaient me faire confiance, alors maintenant, ça ne pose plus de problème." (Cennet, 26 ans, étudiante, Hambourg) Souvent, les parents encouragent leur enfant, car une telle réussite signe l'aboutissement de leur propre projet migratoire. Mais, parfois, le rapport au reste de la population turque est tendu, notamment pour les jeunes filles. Ces dernières peuvent être l'objet de jalousie, d'invectives et de jugements moraux.

\section{Une autonomie parfois difficile}

La situation d'Ipek à Bordeaux illustre la difficulté que peuvent ressentir certaines filles turques dans leur conquête d'une telle autonomie. Ipek est étudiante dans une filière scolaire sélective et durant sa scolarité, au collège et au lycée, la 
jeune femme a eu des résultats scolaires brillants. Pourtant, certains membres de sa famille la découragent dans ses études, en lui expliquant que ces dernières ne servent à rien puisqu'elle est une femme et que, pour cette raison, elle se mariera (supposant par là qu'Ipek n'aura d'autre choix que d'accepter le changement de cap que peut induire le mariage dans la vie d'une jeune femme turque). Au final, la jeune femme ne veut pas se marier, ou uniquement avec un Français, et ne souhaite pas avoir d'enfants. Elle expose très douloureusement son amertume et sa peine, expliquant qu'elle trouve injuste d'être mise au banc de la communauté alors qu'elle est reconnue pour son mérite par tous ses enseignants.

"Ils [les membres de son réseau familial] sont vraiment arriérés et débiles; logique vu qu'ils sont du Millî Görüs ${ }^{(8)}$; tout passe par la religion ; ils veulent que les fermmes, que nous, on accepte gentiment de nous laisser faire."

Globalement, les positions de ces jeunes quant à la place de la religion dans l'existence, le féminisme, l'antisémitisme ou les questions politiques épineuses (kurde, arménienne) ne diffèrent plus de celles de leurs semblables non turcs. Elles reflètent la pensée dominante des sociétés occidentales, véhiculée par les médias. Ces adaptations sociales marquent une conformité revendiquée aux standards sociaux ambiants. Elles expriment l'effectivité d'une dynamique migratoire classique alors que les premières générations turques étaient réputées communautaires. Ces modalités d'intégration sociale ne sont pourtant pas légion, elles coexistent aux côtés de formes plus communautaires d'intégration et de parcours marqués par le paupérisme juvénile urbain.

\section{La revendication communautaire}

En France comme en Allemagne, l'expérience des jeunes issus de l'immigration turque peut aussi se caractériser par la centralité de l'identité ethnique. Cette identité se définit par une assimilation assez faible à la société environnante et par une intégration relativement réussie. Ces jeunes placent leurs pratiques et leurs relations sociales, leurs loisirs et leur engagement associatif, culturel, politique, dans le cadre des normes et des espaces communautaires turcs. Ils se considèrent Turcs et voient la société majoritaire comme étrangère sans qu'il n'existe de tensions à son égard. Le groupe ethnique est ici un vecteur essentiel de socialisation, d'insertion et de construction identitaire. Les jeunes placés dans ce schéma affirment leur volonté de perpétuer le modèle de la communauté ethnique construit par leurs aînés. Pourtant, l'expression de ces identités ne se fait pas de la même manière en France et en Allemagne, parce que l'état et la forme des communautés turques diffèrent 
dans ces deux espaces. À Bordeaux, les jeunes générations adaptent à leur contexte juvénile ambiant le dynamisme communautaire des Turcs. À Hambourg, au contraire, les jeunes assistent malgré eux davantage à la décomposition de l'espace ethnique. Dans les deux cas, ces identités marquent la revendication du désir de rester membre d'une Turquie de l'extérieur ${ }^{(9)}$.

\section{Hambourg : identité nationale contre dilution des repères culturels}

À Hambourg, certains piliers économiques et associatifs turcs se décomposent dans le tissu urbain. Ils deviennent donc de moins en moins visibles et opératoires pour les jeunes. Un facteur démographique participe à cette dilution. La force numérique des Turcs à Hambourg (près de 60000 individus) produit une diminution de l'interconnaissance interne à la communauté. Cela rend les différentes organisations turques moins repérables donc moins investies, ce qui les affaiblit. Un autre facteur est endogène à l'immigration turque : à Hambourg, les ressortissants turcs présentent une origine socio-économique et spatiale plus variée qu'à Bordeaux. Il existe donc une plus grande diversité de l'offre politique, culturelle et religieuse sur le "marché" organisationnel turc. Cette concurrence des repères identitaires contribue ainsi à détendre l'univers ethnique et encourage l'affirmation individualiste.

Par ailleurs, l'entretien d'une solidarité collective est fonction du contexte interethnique. À Hambourg, la minorité turque, en situation de minorité majoritaire, est le premier réceptacle de la stigmatisation. Elle ne peut donc pas construire des stratégies de distinction (et d'invisibilisation) à l'égard d'une autre minorité, stratégie utilisée par les Turcs à Bordeaux à l'égard des personnes issues des migrations postcoloniales.

Les premiers Turcs s'installent à Hambourg dès le début des années soixante (accords économiques bilatéraux) et sont employés individuellement dans l'économie locale (industries, activités portuaires, chemin de fer). Le besoin de réseaux ethniques collectifs ne se faisait alors pas sentir. Ces réseaux n'ont donc pas été construits et ne peuvent pas servir d'alternatives à l'exclusion des jeunes générations. De facto, les jeunes souhaitant s'intégrer de manière communautaire ne peuvent le faire. Ces jeunes sont donc en passe d'acculturation : ils perdent la maîtrise de la langue turque, sont moins présents dans les espaces turcs de socialisation et se tournent moins vers des registres ethniques de mobilité sociale. Ils vivent donc une situation de dissonance entre leur souhait de se définir comme 
Turc de l'extérieur et la trop faible opérationnalité de l'espace ethnique pour la construction de cette identité. Cette position génère un tâtonnement identitaire. L'absence d'éléments concrets permettant de perpétuer l'identité turque exilée semble régulée par le développement d'une idéologie nationale abstraite et militante. Par exemple, leurs propos font mention d'épisodes glorieux de l'histoire natio-

Ces jeunes sont donc en passe d'acculturation : ils perdent la maîtrise de la langue turque, sont moins présents dans les espaces turcs de socialisation et se tournent moins vers des registres ethniques de mobilité sociale. nale (kémalisme, période ottomane). Ils évoquent le mythe d'une Grande Turquie, le $\operatorname{Turan}^{(10)}$, comme paradis perdu, alors que cette notion semble totalement désuète et devrait a priori peu mobiliser des jeunes ayant grandi en Europe occidentale. Certains affichent d'ailleurs leur sympathie pour l'extrême droite turque (Loups gris, MHP). Il semble donc que l'effritement de la communauté en tant qu'espace social concret produise, chez certains jeunes, un surinvestissement dans le registre idéologique abstrait. À Bordeaux, la situation est différente : la communauté se maintient et les jeunes participent à son adaptation. Cela renforce une identité communautaire et villageoise positive.

\section{Bordeaux : l'adaptation ethnique}

À Bordeaux, les ressources communautaires (entrepreneuriat turc, réseaux social et associatif) apparaissent plus opératoires et plus visibles, ce qui alimente un entre-soi et le noyau dur ${ }^{(1)}$ de l'identité turque (l'islam turc, hanéfite, et le nationalisme turc, la turcité). Ces jeunes maintiennent le lien avec la culture villageoise d'origine et s'investissent dans des associations culturelles ou dans les mosquées du DITIB ${ }^{(12)}$. Cela entretient une maitrise relativement bonne de la langue turque. Idéologiquement, ces jeunes se tiennent éloignés des valeurs turques les plus radicales ; l'existence concrète d'une communauté semble rendre superflu l'investissement dans les thèses ultranationalistes. "Nous sommes Turcs et [...] solidaires [...] on est comme une famille [...] nous, on n'est pas des racistes ou des très religieux, on a notre famille, notre tradition, ça suffit bien." (Hulya, lycéenne, 17 ans)

Dans le domaine économique, l'entrepreneuriat turc du bâtiment offre des débouchés professionnels. Nombre de jeunes garçons travaillent au moins occasionnellement dans la construction. Certains d'entre eux ont créé leur entreprise et 
connaissent de vrais succès ${ }^{(13)}$. Umit a par exemple fondé une entreprise dans le bâtiment alors que ses résultats scolaires ne l'autorisaient pas à accéder aux filières générales. Bénéficiant des dispositifs d'allègement de charges sociales, son entreprise est aujourd'hui en expansion et emploie plus d'une dizaine de jeunes compatriotes. Sa réussite professionnelle sert d'exemple au sein de la communauté dans laquelle il est très bien intégré.

L'expansion du secteur de la construction a permis de proposer des emplois plus qualifiés (conduite de travaux, architecture) et féminisés (comptabilité, gestion, secrétariat). En conséquence, nombre de jeunes, comme Umit, ne pouvant pas accéder aux filières scolaires d'excellence, orientent leur scolarité vers le marché turc du travail, celui-ci étant protégé. Ils ont ainsi une place dans la division du travail. À Bordeaux, l'existence de ressources solides permet à l'identité ethnique de se construire positivement et paisiblement, à l'abri des idéologies radicales. Cette appartenance est entretenue et revendiquée pour se prémunir de la relégation urbaine subie par nombre de jeunes issus des migrations postcoloniales. La catégorie des jeunes de banlieue exerce une fonction symbolique importante dans les quartiers défavorisés. Si pour ces jeunes intégrés dans la communauté turque, elle est un repoussoir, elle suscite aussi le mimétisme pour d'autres jeunes d'ascendance turque, pourtant jusqu'ici réputés "sans histoire".

\section{Les impasses de la condition minoritaire}

En France et en Allemagne, certains jeunes ont été embarqués dans la "galère" caractérisant les quartiers d'exil ${ }^{(14)}$. Une telle attraction s'explique par l'éloignement de l'espace familial et communautaire traditionnel, conjugué à l'insuffisance de ressources légitimes (scolaires notamment) pour la production de parcours autonomes et choisis. Dans de telles conditions, ces parcours valident la constitution de secondes générations déclinantes ${ }^{(15)}$, caractérisées par leur pénétration dans l'underclass urbaine. Généralement, l'éloignement de la communauté est réciproque : la communauté villageoise se désolidarise de ces jeunes, par honte ou délégitimation. Cette distance signe une certaine forme d'assimilation; pourtant, celle-ci ne se fait pas au niveau du standard dominant de la classe moyenne, mais à l'égard de l'univers directement environnant, relégué et paupérisé. La condition minoritaire ${ }^{(16)}$ s'illustre donc par une assimilation assez forte (langue, modes de consommation, relations amoureuses, souhaits de réussite sociale) et par une absence d'intégration économique. Alors que les ambitions sociales de ces jeunes les rapprochent de leurs homologues non turcs et que les référents 
culturels turcs se perdent, tout porterait à croire qu'un processus d'assimilation légitime soit à l'ceuvre. Mais cette dynamique n'opère pas, car elle reste soumise au manque d'intégration économique. Pour combler la dissonance assimilation/absence d'intégration - qui brise la promesse républicaine : assimilation contre égalité -, ces jeunes utilisent les codes sociaux et culturels qui leur sont quotidiennement proposés, ceux de la rue, comme modes de réaction à leur domination sociale ${ }^{(17)}$.

Ici, l'identité intermédiaire construite par ces jeunes est donc réactive : elle répond à l'injustice dont ils expliquent être victimes. Cette identité se base sur une ethnicité, mais celle-ci n'est plus spécifiquement turque; elle affirme plus généralement une altérité à l'égard de la société qui n'intègre pas. Elle est associée à l'islam, qui n'est plus non plus strictement turc (sunnite, hanéfite), mais globalisé pour servir de symbole de la condition minoritaire ${ }^{(18)}$. Enfin, cette identité s'appuie sur l'appartenance ambivalente au quartier de résidence. Le cas de Burak illustre cette dynamique. Le jeune homme a été exclu de son lycée professionnel en raison de son absentéisme et de son insolence. Il est vêtu de survêtements et de baskets et arbore fièrement un pendentif représentant le drapeau turc. Burak passe le plus clair de son temps libre dans les cages d'escalier des tours de son quartier. Ses parents et le reste de la communauté désapprouvent son évolution et le rejettent. Burak ne se rend plus dans les fêtes et les lieux communautaires, juge "bidons" les emplois proposés par les entreprises turques et se plaît à évoquer ostensiblement son attrait pour le rap, la Turquie, l'islam et sa cité.

Ici, les critères d'altérité (religion, ethnicité, quartier) perdent la référence à la communauté d'origine (villageoise). Ils sont élargis pour concerner le plus grand nombre de jeunes vivant dans les grands ensembles. Le but est de construire un dénominateur commun à une "ethnicité de la misère", se définissant par sa domination à la société majoritaire. Ce faisant, ces jeunes s'approprient la différence que la société leur renvoie et qui alimente leur absence d'intégration. Il s'agit d'un retournement du stigmate, par lequel un cercle vicieux de mise à l'écart se maintient.

\section{Un ressentiment plus violent en France}

Dans les deux scènes nationales, cette identité réactive s'exprime contre les institutions, dont les pratiques sont décrites comme discriminatoires, particulièrement celles de la police et de l'école. Cela étant, cette identité se décline différemment en France et en Allemagne. L'amertume et les sentiments d'injustice s'expriment plus forte- 
ment en France et les rapports sociaux y semblent plus violents. En Allemagne, les rapports police/jeunes minoritaires ne sont pas aussi tendus qu'en France. Il n'y a pas non plus d'émeutes, bien que les quartiers marginalisés aient conjoncturellement leur lot d'objets et d'engins incendiés. Aucun jeune Allemand n'a mentionné avoir été victime de violences policières, verbale ou physique. En Allemagne, l'école est sévèrement critiquée par ces jeunes : pour eux, elle a été ce qui a légitimé la fracture ethnique entre eux et les Allemands. Mais ces reproches restent limités par rapport au cas français, alors que la situation des jeunes issus de l'immigration est bien plus préoccupante en Allemagne qu'elle ne l'est en France.

C'est donc dans le pays réputé universaliste que l'on se trouve le plus, lorsque l'on est minoritaire, en situation de rapport de force violent et douloureux avec les institutions. Ce paradoxe surprend. L'Allemagne s'illustre davantage par des pratiques juridiques et scolaires différentialistes ${ }^{(19)}$. Elle a aussi connu des actes de racisme sans équivalent en France (Solingen, Mölln). Pourtant, ce pays matérialise des rapports sociaux plus apaisés qu'ils ne le sont en France.

\section{Conclusion}

Les constructions identitaires des jeunes issus de l'immigration dépendent de figures d'intégration, elles-mêmes fonctions des rapports entre l'intégration et l'assimilation à la société majoritaire et de la configuration des relations entretenues avec la communauté, la cité et la société environnante. Les tendances fortes de ces parcours d'intégration (communes aux deux pays) ne dépendent ni directement de la population turque elle-même ni des dispositifs institutionnels, mais de l'évolution de l'immigration dans les grandes villes d'Europe occidentale. Les inflexions de ces tendances semblent témoigner des relations plus spécifiques que les deux États entretiennent avec leurs minorités. Ces inflexions marquent les différences entre les jeunes d'ascendance turque en France et en Allemagne. En France, le maintien plus fort d'une communauté dans un contexte marqué par l'universalisme républicain et la présence de rapports sociaux plus durs entre minoritaires et institutions censées être égalisatrices en sont des exemples. Il existe donc une dissonance entre les philosophies des États et les conditions objectives des acteurs, de même qu'il existe un écart entre ces conditions objectives et les subjectivités qui en découlent. In fine, les acteurs croient ce que les modèles leur promettent. Plus l'écart entre la norme philosophique et la réalité sociale est grand, plus les amertumes et les frustrations accentuent leur expression. 


\section{Notes}

1. Le sujet de la jeunesse issue de l'immigration, associé à celui de l'insécurité urbaine et du chômage, s'impose souvent dans le débat politique et médiatique. La campagne présidentielle française de 2002 et la campagne de Roland Koch de 2008 en Hesse en sont deux exemples manifestes.

2. En France, selon l'INED, le "cas turc" serait le plus communautaire, le moins assimilé ; il serait une exception au modèle républicain d'intégration.

3. Stéphane de Tapia, "Le champ migratoire turc et l'Europe", Enjeux de l'immigration turque en Europe, Paris, L'Harmattan-CIEMI, 1995.

4. Isabelle Rigoni, Mobilisations, actions et recompositions. Migrants de Turquie et réseaux associatifs en France, en Allemagne et en Belgique, thèse de doctorat, 2000.

5. Michèle Tribalat, De l'immigration à l'assimilation, Paris, La Découverte, 1996 ; Michèle Tribalat, Une enquête sur les immigrés et leurs enfants, Paris, La Découverte, 1995 ; Roger Establet, Comment peut-on être Français ?, Paris, Fayard, 1997 ; Françoise Rollan et Benoît Sourou, Les migrants turcs de France. Entre repli et ouverture, Pessac, MSHA, 2006.

6. Alejandro Portes, Min Zhou, "The New Second Generation : Segmented Assimilation and Its Variants", Political and Social Science, vol. 530, nov. 1993 ; Roxane Silberman et Irène Fournier, "Les secondes générations sur le marché du travail en France : une pénalité ethnique ancrée dans le temps. Contribution à la théorie de l'assimilation segmentée", Revue française de sociologie, vol. 47, n², 2006.

7. François Dubet, Immigration, qu'en savons-nous? Notes et études documentaires, La documentation française, $n^{\circ} 4887,1989$.

8. Satellite dans les pays d'immigration du Parti islamiste dur (actuellement Saadet Partisi) d'Erbakan Millî Görüs. À Bordeaux, il est investi au départ par des ressortissants des régions de la mer Noire, mais, depuis peu, l'organisation gagne en popularité, notamment chez les jeunes générations.

9. La "Turquie de l'extérieur" fait référence aux communautés transnationales turques dans les pays d'immigration. Ces espaces sont structurés culturellement, politiquement et économiquement, et fonctionnent de manière autonome par rapport aux sociétés d'implantation et à la Turquie.

10. Le terme "Turan" est d'origine iranienne. Il a d'abord désigné les régions perses du nord et de l'est, puis le "foyer primitif" supposé en Asie centrale des peuples de "race touranienne", amalgame des peuples ouralo-altaïques et finnoougriens. Le touranisme, expression idéologique et politique de l'unité des peuples, s'est diffusé chez les théoriciens du nationalisme turc comme Ö. Seyfeddin, T. Alp, Z. Gökalp. Chez eux, Turan désigne l'État futur qui résultera de l'union des peuples turcs et touraniens. Voir François Georgeon, Aux origines du nationalisme turc, Paris, ADPF, 1980, p. 29-30.

11. Riva Kastoryano, Être Turc en France. Réflexions sur familles et communauté, Paris, L'Harmattan, 1987 ; Riva Kastoryano, "Être Turc en France et en Allemagne", in Enjeux de l'immigration turque en Europe, Paris, L'HarmattanCIEMI, 1995 ; Dominique Schnapper, L'Europe des immigrés. Essai sur les politiques d'immigration, Paris, Bourin, 1992.

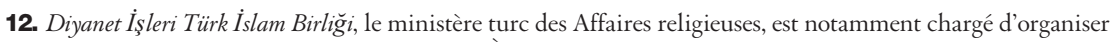
le culte des ressortissants de Turquie en Europe. À Bordeaux, les adhérents du DITIB ont une lecture plus libérale de l'islam que leurs homologues du Millî Görüs.

13. Nombre de "talents des cités" régionaux sont Turcs et un des récents "talents" nationaux est Turc, chef d'une entreprise d'électricité prospère.

14. François Dubet, La galère. Jeunes en survie, Paris, Fayard, 1987 ; François Dubet et Didier Lapeyronnie, Les quartiers d'exil, Paris, Seuil, 1999.

15. Herbert J. Gans, "Second Generation Decline: Scenarios for Economics and Ethnic Futures of the post 1965 American Immigrants", Ethnic and Racial Studies, vol. 15, n², april 1992.

16. Didier Lapeyronnie, "Les deux figures de l'immigré", in Michel Wieviorka, Une société fragmentée? Le multiculturalisme en débat, Paris, La Découverte, 1997.

17. Daniel Lepoutre, Cceur de banlieue. Codes, rites et langages, Paris, Odile Jacob, 2001.

18. Des garçons et des filles s'engagent par exemple dans des formes militantes de religiosité véhiculées par des organisations et des confréries islamiques. Ces situations existent depuis peu et demeurent marginales par rapport aux autres figures d'identité.

19. Rogers Brubaker, Citoyenneté et nationalité en France et en Allemagne, Paris, Belin, 1996 ; Pierre Weil, Qu'est-ce qu'un Français?, Paris, Grasset, 2002. 